\title{
An extraordinary stringent and sensitive light-switchable gene expression system for bacterial cells
}

Cell Research (2016) 26:854-857. doi:10.1038/cr.2016.74; published online 17 June 2016

Dear Editor,

Light-switchable gene expression systems provide transient, non-invasive and reversible means to control biological processes with high tunability and spatiotemporal resolution. In bacterial cells, a few light-regulated gene expression systems based on photoreceptors and two-component regulatory systems (TCSs) have been reported, which respond to blue, green or red light [15]. These systems have already been used for a number of synthetic biology studies. Particularly, sophisticated temporal control of gene expression with unparalleled accuracy and precision can be achieved using these systems [1, 3-5]. However, their widespread usage is limited by a few drawbacks: limited portability due to multiple components [1-5], dependence on extraneous light-sensitive cofactors and appropriate cofactor synthesis genes $[1,3-5]$, low yield of recombinant proteins due to weak induction efficiencies (much less than $\mathrm{T} 7$ expression systems) [1-5], lethal effect when gene product is toxic and difficulties in studying low-abundance proteins due to high leakage under the non-inducing conditions [15]. In addition, light-sensitive two-component systems are hardly adaptable to different promoters, as the gene regulation occurs only with specific cognate response regulator and promoter [1-5]. An ideal light-switchable gene expression system should be simple and easy to manipulate, and has high induction efficiency, low leakage, high sensitivity toward light, low toxicity, and low interference with endogenous proteins or genes [6].

Previously, we reported a simple yet robust transgene system to spatiotemporally control mammalian cell gene expression based on a single synthetic, genetically encoded light-switchable transactivator [7]. We sought to create a highly efficient light-switchable repressor for bacterial cells based on a similar design. To this end, we fused the blue light sensor VIVID (VVD), a small light-oxygen-voltage (LOV) domain-containing protein from Neurospora crassa [8], to the C-terminus of DNA-binding domain of LexA repressor of the Escherichia coli SOS regulon [9]. Upon blue light irradiation, a cysteine-flavin adduct is formed in the VVD domain, causing conformational changes of the domain and the subsequent dimerization of the fusion protein $[8,10]$. The activated protein dimer would then bind its cognate operator sequence and repress the promoter activity (Figure 1A). After screening the linker between LexA and VVD, we obtained a mutant that manifested $\sim 10$ 000-fold repression of ColE promoter-driven reporter gene expression in JM109 (DE3, AsulA, LLexA) cells illuminated with weak blue light $\left(4.9 \mathrm{~W} / \mathrm{m}^{2}, 460 \mathrm{~nm}\right.$ LED lamp; Figure 1B, Supplementary information, Figure $\mathrm{S} 1 \mathrm{~A}$ and $\mathrm{S} 1 \mathrm{~B})$. We referred to the light-switchable repressor LexA-VVD as LEVI and the gene expression system based on it as the LightOff system. The LightOff system has high induction efficiency similar to T7-driven pET expression system, yet with much lower leakage (Figure 1B).

For the LightOff system, light-induced shutdown of transcription of target genes occurred rapidly, as shown by the sharp decrease of target gene mRNA levels and the stop of protein production in the cells after blue light illumination (Figure 1C and Supplementary information, Figure S1C). The drop of target protein level was significantly slower than the drop of mRNA level, as it takes time for the protein already synthesized to be degraded and diluted during cell growth. When cells were transferred to darkness, LEVI was inactivated and dissociated from the DNA, relieving the transcriptional repression of the target gene and restoring protein production. In these cells, the mRNA levels of target genes increased by $>10000$-fold $2.5 \mathrm{~h}$ after the stop of blue light illumination, while it took longer for the protein levels to recover (Supplementary information, Figure S1C-S1E). We found that in batch culture studies, the derepression kinetics of the LightOff system was significantly faster than that of the pDusk system (Supplementary information, Figure S1D), a blue light-regulated TCS-based gene expression system [2]. The derepression of the LightOff system was markedly accelerated when cells were kept in exponential growth (Supplementary information, Figure S1E). Under the exponential growth condition, the 
A

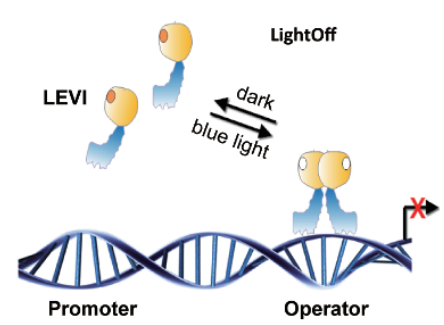

D

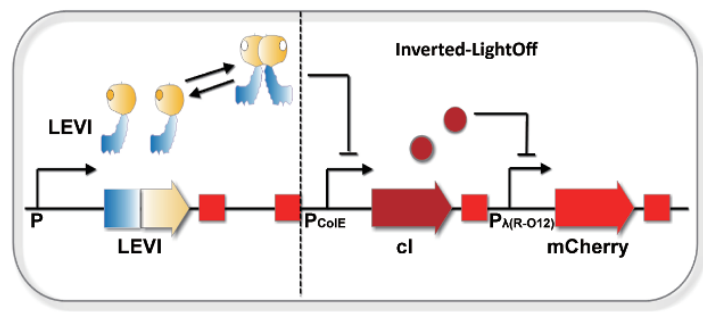

B

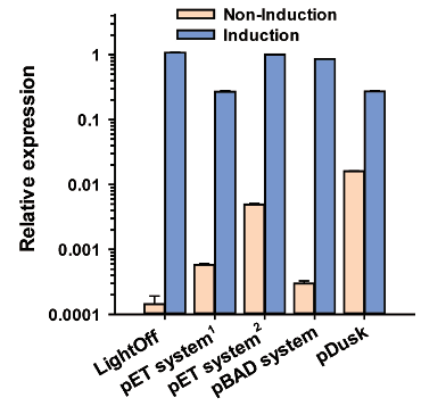

E
C

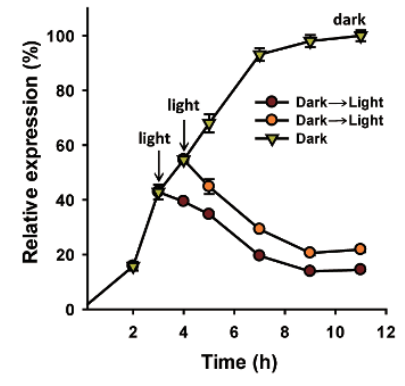

F
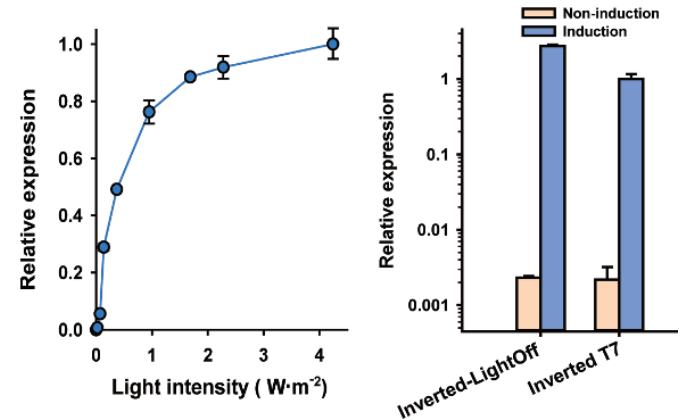

G

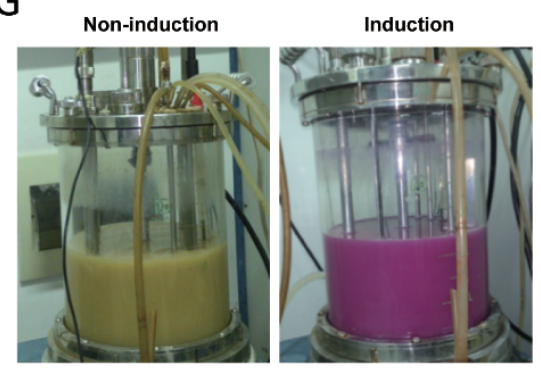

H

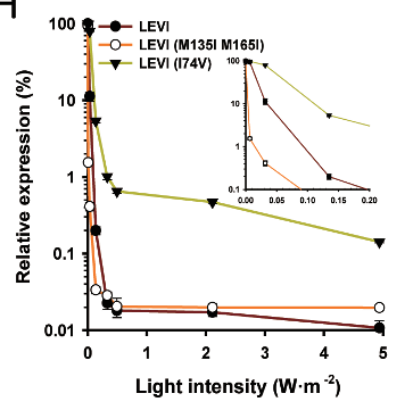

I

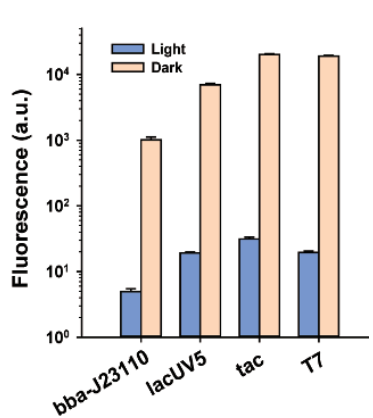

Figure 1 The LightOff gene expression system. (A) Schematic representation of the LightOff system. Upon light exposure, the light-switchable repressor LEVI is induced to form homodimers, which then bind to the operator sequence and repress the activity of promoter by blocking the attachment of RNA polymerase to the promoter. Removal of blue light results in gradual dissociation of the dimers and transcription activation. (B) Comparison of gene expression regulation by various chemical- or light-regulated systems. mCherry expression regulated by the LightOff system in JM109 (DE3, $\triangle$ sulA, $\Delta$ LexA) cells and pDusk system in BL21 (DE3) cells was analyzed $18 \mathrm{~h}$ after cells were cultured with light illumination (non-induction) or in darkness (induction). mCherry expression regulated by pET system in BL21 (DE3) pLysS (pET system ${ }^{1}$ ) or BL21(DE3) (pET system ${ }^{2}$ ) cells and pBAD system in TOP10 cells was analyzed $18 \mathrm{~h}$ after cells were cultured without (non-induction) or with addition of chemical inducers (induction). Data shown were normalized to mCherry expression level in BL21(DE3) cells with the pET system in the presence of chemical inducer. (C) Fast repression kinetics of LightOff system in batch culture. JM109 (DE3; $\triangle$ sulA $\triangle L e x A)$ cells transformed with LightOff system were cultured in darkness and then transferred to blue light at the indicated time points (black arrow). Data were normalized to the mCherry expression level in dark conditions. (D) Schematic representation of the Inverted-LightOff system. cl repressor, which can repress the activity of R-O12 promoter, is under the control of the LightOff system. Upon light illumination, LEVI homodimerizes, represses the expression of cl repressor and initiates target gene expression. (E) Quantitative control of gene expression by modulating the light irradiance in the Inverted-LightOff system. Data were normalized to the maximal expression level upon light irradiance. (F) Comparison of the performance of the Inverted-LightOff system with inverted T7 system. Data were normalized to mCherry expression level induced by the inverted T7 system without IPTG. (G) 5-L bioreactor for large-scale production of recombinant protein by the LightOff system. (H) Light sensitivity of the LightOff system with different VVD mutations. Data were normalized to mCherry expression levels in cells kept in darkness. Inset shows the enlarged region of low light intensities. (I) Adaptability of LEVI in controlling transcriptional activities of various promoters. LexA cognate operator sequence was incorporated into various constitutive promoters, bba-J23110, lacUV5, tac and T7 promoters. Detection of the activities of these promoters upon blue light irradiance or in darkness was conducted in BL21 (DE3) cells. a.u., arbitrary units. Data shown in all bar graphs represent mean \pm SEM from three independent experiments. 
switching time of protein induction, i.e., the time it takes for protein level to reach its $50 \%$ maximal expression level, was $3 \mathrm{~h}$. The switching time of protein induction is $40 \mathrm{~min}$ for the Cph8-OmpR system and $44 \mathrm{~min}$ for the CcaS-CcaR system [5]. Therefore, it takes a longer time for the LightOff system to switch from fully repressed state to fully induced state, compared with the CcaS-CcaR and Cph8-OmpR systems. Nevertheless, the LightOff system can still rapidly switch on and off gene expression (Supplementary information, Figure S1F), and thus may be used similarly to the CcaS-CcaR and Cph8-OmpR systems to temporally regulate gene expression [5]. The LightOff system may become a beneficial addition to previous systems in even more sophisticated gene circuit programming [5], as these systems are regulated by different colors of light. Two sets of genes may be regulated independently in the same cell using the combination of the LightOff system and the CcaS-CcaR or Cph8-OmpR system.

The ability of the LightOff system to spatially control gene expression was examined using a plate-based assay similar to a previous report [1]. The bacterial lawns covered by a specific photomask were exposed to blue light. The subsequent fluorescence imaging showed an mCherry expression pattern that precisely reflected the shape of the photomask (Supplementary information, Figure S1G-S1I). The LightOff system can be transformed into a light-activated gene expression system (termed the Inverted-LightOff system) when a cI/P ${ }_{\lambda(\mathrm{R}-\mathrm{O} 12)}$ "NOT" gate gene circuit was added (Figure 1D). The Inverted-LightOff system displayed excellent light-dependent gene activation (Figure 1E) with $>1000$-fold induction efficiency, which outperformed chemical-inducible inverted T7 system (Figure 1F). It should be noted that the light sensitivity of the Inverted-LightOff system is far less than that of the LightOff system, as the expression of cI repressor must be fully repressed by high-intensity light to activate the mCherry gene expression. The light sensitivity of Inverted-LightOff system may be further improved by fine-tuning the activity of the promoter upstream of the cI repressor. Furthermore, we explored the feasibility of application of the LightOff system in LexA WT strains. We modified the system by introducing mutations to both LexA DNA-binding moiety of LEVI and the cognate operator sequence [9] to avoid the interference of endogenous wtLexA protein and operator regions in bacterial SOS signal pathway. The modified system could efficiently switch gene expression irrelevant to the presence of wtLexA (Supplementary information, Figure S1J).

The extremely stringent regulation of the LightOff system can be useful for many quantitative and synthetic biology studies, as well as expression of toxic proteins. For example, CcdB, a protein lethal to Escherichia coli (Supplementary information, Figure S2A), can be produced using the LightOff system (Supplementary information, Figure S2B). Quantitative expression of cheZ, which plays an important role in bacterial chemotaxis [11], by the LightOff system in cheZ-deleted bacteria restored light irradiance-regulated mobility of these bacterial cells (Supplementary information, Figure S2C and S2D). Directed mobility of the "phototactic" bacteria was also observed when using a printed mask with a specific image as the "light guide" (Supplementary information, Figure S2E). LEVI exhibits extraordinary sensitivity to light, which is critical for prokaryotic light-switchable gene expression systems, as bacterial cells are often cultured in high density $\left(\mathrm{OD}_{600} \sim 2-100\right)$, making light difficult to penetrate. Our data showed that the LightOff system can be applied successfully for large-scale production of recombinant proteins by high-density $\left(\mathrm{OD}_{600}\right.$ 20) fermentation using 5-L bioreactor (Figure $1 \mathrm{G}$ and Supplementary information, Figure S2F), which has not been demonstrated by any other prokaryotic light-switchable gene expression system. Such ability of the LightOff system is not only convenient but also cost-saving compared with chemical inducers. Owing to LEVI's modular design, it is possible to further enhance or reduce the light sensitivity of LightOff system by introducing VVD mutations in LEVI to alter the duration of light-activated states. The gene expression repression by LEVI (Supplementary information, Figure $1 \mathrm{H}$ ) fits well by a Hill function (Hill coefficient $n=1.07 \pm 0.05$ ) with a light sensitivity (half-maximal response, $k$ ) of $2.6 \mathrm{~mW} / \mathrm{m}^{2}$ (Supplementary information, Table S1A). LEVI with M135I M165I double mutation in its VVD moiety, which slows the decay of light-activated state [12], exhibited extreme sensitivity to blue light (half-maximal response is 0.71 $\mathrm{mW} / \mathrm{m}^{2}$ ), and $6.5 \mathrm{~mW} / \mathrm{m}^{2}$ weak light could already result in $\sim 60$-fold repression of gene expression (Figure $1 \mathrm{H}$ and Supplementary information, Table S1A). On the contrary, I74V mutation of VVD, which greatly accelerates the decay of light-activated state [12], made LEVI much less sensitive to light (Figure 1H and Supplementary information, Figure S2G), but with faster derepression kinetics when light was shut down (Supplementary information, Figure S1D and S1E).

LEVI is the only single-component light-switchable repressor to date, which shall reduce the possibility of interaction with the host chassis. LEVI can be used to control transcriptional activities of various promoters, in which LexA cognate operator sequence is incorporated (Figure 1I and Supplementary information, Data S1), illustrating the adaptability of LEVI. Therefore, LEVI may 
be used to control gene expression in other bacteria and even in eukaryotic cells.

The LightOff system has many favorable characteristics, such as single component, high induction efficiency, extremely low leakage and precise spatiotemporal resolution (Supplementary information, Table S1B), enabling it to be a powerful and convenient tool for study of bacterial gene function and gene regulatory networks and for large-scale production of recombinant protein. Taken together, our data indicate that the LightOff system is a robust and versatile tool for rapid, reversible, quantitative and spatiotemporal control of gene expression in bacteria.

\section{Acknowledgments}

This work was supported by the National Natural Science Foundation of China (31225008, 91313301, 31170815 and 31470833), Shanghai Science and Technology Commission (12JC1402900 and 11DZ2260600), Dawn Program of the Shanghai Education Commission (11SG31), State Key Laboratory of Bioreactor Engineering, the 111 Project (B07023) and the Fundamental Research Funds for the Central Universities.

Xianjun Chen ${ }^{1,2,3}$, Renmei Liu ${ }^{1,2}$, Zhengcai Ma ${ }^{1}$, Xiaopei $\mathrm{Xu}^{1,2}$, Haoqian $\mathrm{Zhang}^{4}$, Jianhe $\mathrm{Xu}^{1}$, Qi Ouyang ${ }^{4}$, Yi Yang ${ }^{1,2,3}$
${ }^{I}$ Synthetic Biology and Biotechnology Laboratory, State Key Laboratory of Bioreactor Engineering, Shanghai Collaborative Innovation Center for Biomanufacturing Technology, East China University of Science and Technology, 130 Mei Long Road, Shanghai 200237, China; ${ }^{2}$ Optogenetics and Molecular Imaging Interdisciplinary Research Center, CAS Center for Excellence in Brain Science, East China University of Science and Technology, 130 Mei Long Road, Shanghai 200237, China; ${ }^{3}$ Collaborative Innovation Center of Genetics and Development, East China University of Science and Technology, 130 Mei Long Road, Shanghai 200237, China; ${ }^{4}$ State Key Laboratory for Mesoscopic Physics and School of Physics, Peking University, Beijing, China

Correspondence: Yi Yang

E-mail: yiyang@ecust.edu.cn

\section{References}

1 Levskaya A, Chevalier AA, Tabor JJ, et al. Nature 2005; 438:441-442.

2 Ohlendorf R, Vidavski RR, Eldar A, et al. J Mol Biol 2012; 416:534542.

3 Tabor JJ, Levskaya A, Voigt CA. J Mol Biol 2011; 405:315-324.

4 Schmidl SR, Sheth RU, Wu A, et al. ACS Synth Biol 2014; 3:820-831.

5 Olson EJ, Hartsough LA, Landry BP, et al. Nat Methods 2014; 11:449455.

6 Keyes WM, Mills AA. Trends Biotechnol 2003; 21:53-55.

7 Wang X, Chen X, Yang Y. Nat Methods 2012; 9:266-269.

8 Zoltowski BD, Schwerdtfeger C, Widom J, et al. Science 2007; 316:1054-1057.

9 Thliveris AT, Little JW, Mount DW. Biochimie 1991; 73:449-456.

10 Zoltowski BD, Crane BR. Biochemistry 2008; 47:7012-7019.

11 Sanna MG, Simon MI. J Bacteriol 1996; 178:6275-6280.

12 Zoltowski BD, Vaccaro B, Crane BR. Nat Chem Biol 2009; 5:827-834.

(Supplementary information is linked to the online version of the paper on the Cell Research website.) 